\title{
Uniaxial tension test on Rubber at constant true strain rate
}

\author{
J.-C. Petiteau ${ }^{1}$, R. Othman ${ }^{1}$, E. Verron ${ }^{1}$, H.L. Sourne ${ }^{2}$, J.-F. Sigrist ${ }^{4}$, and G. Barras ${ }^{3}$ \\ ${ }^{1}$ LUNAM Université, École Centrale de Nantes, GeM, UMR CNRS 6183, BP. 92101, 44321 Nantes Cedex 3, \\ France \\ ${ }^{2}$ LUNAM Université, Institut Catholique des Arts et Métiers (ICAM), 35 avenue du champ de Manœuvres, \\ 44470 Carquefou, France \\ ${ }^{3}$ DGA Techniques Navales, Avenue de la Tour Royale, BP. 40915, 83050 Toulon Cedex, France \\ ${ }^{4}$ DCNS BU Propulsion, Indret, 44620 La Montagne, France
}

\begin{abstract}
Elastomers are widely used for damping parts in different industrial contexts because of their remarkable dissipation properties. Indeed, they can undergo severe mechanical loading conditions, i.e., high strain rates and large strains. Nevertheless, the mechanical response of these materials can vary from purely rubber-like to glassy depending on the strain rate undergone. Classically, uniaxial tension tests are made in order to find a relation between the stress and the strain in the material at various strain rates. However, even if the strain rate is searched to be constant, it is the nominal strain rate that is considered. Here we develop a test at constant true strain rate, i.e. the strain rate that is experienced by the material. In order to do such a test, the displacement imposed by the machine is an exponential function of time. This test has been performed with a high speed hydraulic machine for strain rates between $0.01 / \mathrm{s}$ and 100/s. A specific specimen has been designed, yielding a uniform strain field (and so a uniform stress field). Furthermore, an instrumented aluminum bar has been used to take into account dynamic effects in the measurement of the applied force. A high speed camera enables the determination of strain in the sample using point tracking technique. Using this method, the stress-strain curve of a rubber-like material during a loading-unloading cycle has been determined, up to a stretch ratio $\lambda=2.5$. The influence of the true strain rate both on stiffness and on dissipation of the material is then discussed.
\end{abstract}

\section{Introduction}

Because of their remarkable dissipative properties, elastomers are widely used as damping parts in industry, especially to absorb shock. Indeed, they can undergo severe mechanical loading conditions, i.e. both large strain and strain rates. Nevertheless, because of their viscoelastic nature, the mechanical response of these materials can vary from purely rubber-like to glassy depending on the strain rate undergone, as shown by Yi et al. [1] or Sarva et al. [2].

In this context it is really important to perform experiments to understand how they react depending on the strain rate. At low and high strain rates, the conventional mechanical frames and the Hopkinson bar set-up [3], respectively, are classicaly considered. However, there is no consensus of opinion on the methodology that should be undertaken at intermediate strain rates. Hoo Fatt \& Bekar [4] and Hoo Fatt \& Ouyang [5] proposed a modified Charpy test. The main idea is to achieve homogeneous strain and stress fields as quick as possible by applying a tensile load on both sides of the specimen with the help of a system of pulleys and cables. Roland et al. [6] used the same idea and modify a drop weight machine. These solutions, however interesting, needs a high accuracy on the design of the pulleys and cables in order to achieve an acceptable synchronisation between loads at specimen extremities: the delay between the two loading time must be insignificant compared to the test duration. Recently, Othman et al. [7] proposed a hybrid servo-hydraulic machine for intermediate strain rate testing. Namely, the conventional piezo-electric transducer is replaced by a three-gages instrumented elastic bar in order to capture transient effects. The three strain-gage signals are then analysed by a wave separation technique proposed in Refs. $[8,9]$ and previously applied to the Hopkinson bar set-up $[10,11]$.

In this paper, we are interested in testing rubber-like materials at constant true strain rates. In the intermediate strain rate range, a technique similar to that proposed by Othman et al. [7] is proposed. Moreover, the specimen is designed to rapidly achieve uniform strain and stress states in order to avoid considering corrections as proposed in $[12,13]$.

\section{Method}

\subsection{Material and sample}

The main aim of the experiments is to perform constant true strain rate tension tests for a wide range of strain rate while achieving large strains. As the measurement of strain can not be direct, we can not use a classical regulation loop to prescribe a constant true strain rate in a part of the sample. Therefore, the feed-back loop should be considered in terms of displacement. For this reason, we developed a special sample for the test. Indeed, we can not use classical dumbbell sample because its center will be stretched more than the zone near the grips and, as rubber response is non-linear, we can not know how the strain field would be. Thus, the section of the sample must be constant between the clamps to ensure the best possible homogeneity of both strain and strain rate field (see Fig. 1). 


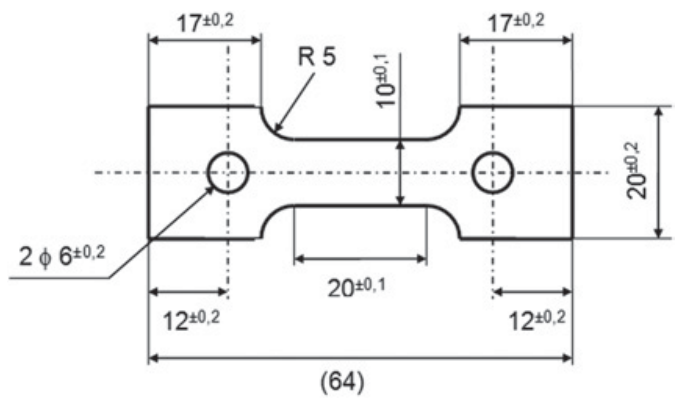

Fig. 1. Design of the sample.

The sample was made of Styrene Butadiene Rubber (SBR) filled with 20phr carbon blacks. The gauge (central part) length is $20 \mathrm{~mm}$. Considering a wave velocity of some hundreds meters per second, the time necessary to achieve homogeneous stress and strain is of some hundreds microseconds. In the most limiting case, i.e., for strain rates about $100 / \mathrm{s}$, the sample is deformed by some percents before homogeneity is achieved in the sample. The sample is deformed less than $1 \%$ before reaching stress and strain homogeneity at a strain rate of about 10/s. The stress and strain in the specimen are assumed homogeneous; and then, the stress is deduced for a boundary force measurement.

\subsection{Machine and loading conditions}

The experimental device is a high speed hydraulic machine (MTS 819). The displacement of the cylinder is controlled by a computer, using a LABVIEW program specially developed to this experiments.

We aim to perform tensile experiments in which the true strain rate tensor $\mathbf{D}$ is kept constant. If we suppose the transformation $\mathbf{x}=\psi(\mathbf{X}, t)$ to be homogeneous in the sample, with a associated deformation gradient $\mathbf{F}$, we can define two different velocity gradient:

- The material one that is based on the initial configuration:

$$
\operatorname{grad} \mathbf{V}(\mathbf{X}, t)=\dot{\mathbf{F}}(\mathbf{X}, t)
$$

- The spatial one that is based on the current configuration that is closer to what really is seen by the material:

$$
\operatorname{grad} \mathbf{v}(\mathbf{x}, t)=\mathbf{L}(\mathbf{x}, t)
$$

The relationship between these two quantities is :

$$
\mathbf{L}=\dot{\mathbf{F}} \mathbf{F}^{-1}
$$

However, this gradient takes into account the rate of rotation and the rate of deformation and so the strain rate tensor is defined as:

$$
\mathbf{D}=\frac{1}{2}\left(\mathbf{L}+\mathbf{L}^{T}\right)
$$

For uniaxial test, the deformation gradient of an incompressible material is:

$$
\mathbf{F}=\left(\begin{array}{ccc}
\lambda & 0 & 0 \\
0 & 1 / \sqrt{\lambda} & 0 \\
0 & 0 & 1 / \sqrt{\lambda}
\end{array}\right)
$$

Table 1. Parameters used for the camera.

\begin{tabular}{|c|c|c|c|}
\hline Strain rate of the test & 0.01 & 10 & 100 \\
\hline Frame frequency (f/s) & 20 & 10000 & 16000 \\
\hline Resolution $\left(\right.$ pixel $\left.^{2}\right)$ & \multicolumn{4}{|c|}{$256 \times 1024$} \\
\hline
\end{tabular}

with $\lambda=l / l_{0}$ the stretch ratio. And so the derivative of the deformation gradient with respect to time is:

$$
\dot{\mathbf{F}}=\left(\begin{array}{ccc}
\dot{\lambda} & 0 & 0 \\
0 & -\frac{\dot{\lambda}}{2 \lambda \sqrt{\lambda}} & 0 \\
0 & 0 & -\frac{\dot{\lambda}}{2 \lambda \sqrt{\lambda}}
\end{array}\right)
$$

Because of the symmetry of the deformation gradient, the strain rate tensor is:

$$
\mathbf{D}=\mathbf{L}=\left(\begin{array}{ccc}
\frac{\dot{\lambda}}{\lambda} & 0 & 0 \\
0 & -\frac{\dot{\lambda}}{2 \lambda} & 0 \\
0 & 0 & -\frac{\dot{\lambda}}{2 \lambda}
\end{array}\right)
$$

As we want $\mathbf{D}$ to be constant, then the stretch ratio has to be : $\lambda=e^{\alpha t}$ instead of $\lambda=\alpha t$ as it is commonly used for tension test at constant engineering strain rate. Then, the deformation gradient is written as:

$$
\mathbf{F}=\left(\begin{array}{ccc}
e^{\alpha t} & 0 & 0 \\
0 & e^{-\frac{\alpha t}{2}} & 0 \\
0 & 0 & e^{-\frac{\alpha t}{2}}
\end{array}\right)
$$

It is though important to notice that for a stretch that is nearly equal to 1 , i.e. for small strain, the two expressions are equivalent. In order to have a constant true strain rate, we control the displacement of the lower grip such as: $u=l_{0}\left(e^{\alpha t}-1\right)$, with $l_{0}$ the initial length of the specimen.

\subsection{Strain and stress measurement}

To measure the strain undergone by the material, a random speckle is painted on the sample. Each test is recorded using a high-speed camera (PHOTRON FASTCAM SA1). The sample must be light with a projector to properly record images. The parameters used for the camera are given in the Table 2. We then use a point tracking technique software (TEMA Automative) to determine the displacement of several points of the recorded images (see Fig. 2). The displacement of those points over time permits to calculate the stretch ratio (and hence the strain rate) in various areas of the sample :

$$
\lambda_{i}(t)=\frac{l(t)}{l_{0}}=\frac{Y_{2}(t)-Y_{1}(t)}{Y_{2}(0)-Y_{1}(0)}
$$

The displacement along the transverse direction is very small and so cannot be properly recorded with the point tracking technique. The displacement over the loading direction (Y) is the only one used to compute the stretch ratio.

As the stress field is assumed homogeneous, an average stress can be calculated for each test from the force measured at an extremity of the specimen. Two force 


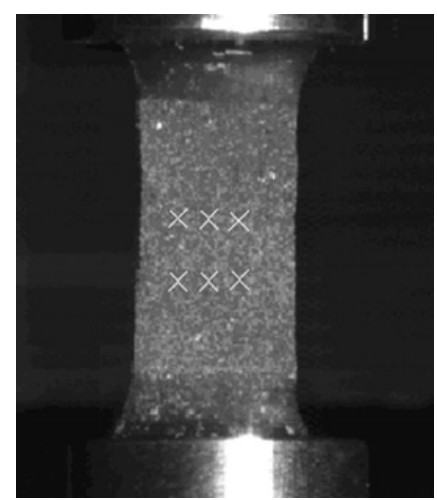

Fig. 2. Position of the points used for tracking.

Table 2. frequencies used for the gauge signals.

\begin{tabular}{|c|c|c|c|}
\hline Strain rate of the test & 0.01 & 10 & 100 \\
\hline Acquisition frequency $(\mathrm{kHz})$ & 0.1 & 100 & 500 \\
\hline
\end{tabular}

measurements are used in our case. The first force measurement is given via a piezoelectric force cell. However, the transient effects are increasingly significant as strain rate increases. Large oscillations are observed on the force signals. Hence, the servo-hydraulic machine is equipped with a second force transducer; it consists in a threestrain-gauges instrumented tubular aluminum bar. This is inspired from the work of Othman et al. [7], although there are two differences. In our case a tubular aluminum bar is used instead of a steel rod in order to increase the signal-to-noise ratio for soft materials. Moreover, the wave separation technique is not considered here. Indeed, the tubular bar is $0.4 \mathrm{~m}$ long and the distance between the gauge is about $0.15 \mathrm{~m}$. Thus, the time for the wave to go from one gauge to the other is about $30 \mu \mathrm{s}$. This is insignificant compared to the test duration. Consequently, the tube can be considered as quasi-statically loaded. The three strain-gauge signals are then averaged before deducing the force applied to the specimen. A schematic picture of the experimental set-up is presented in Fig. 3.

\subsection{Memorandum of the experience}

Five cycles of tension up to a stretch ratio of $\lambda=2.6$ at a strain rate of $0.01 / \mathrm{s}$ were performed the day before to remove the Mullins effect. After placing the painted sample in the grips, we measure the length between grips, that is the initial length of the specimen and input it in the LABVIEW program with the desired strain rate. The displacement over time curve is calculated by the LABVIEW program and the test is started.

\section{Results}

\subsection{Strain}

For each test, we obtain the stretch ratio at different position of the sample (see Fig. 2). The results for $\alpha=1 / \mathrm{s}$

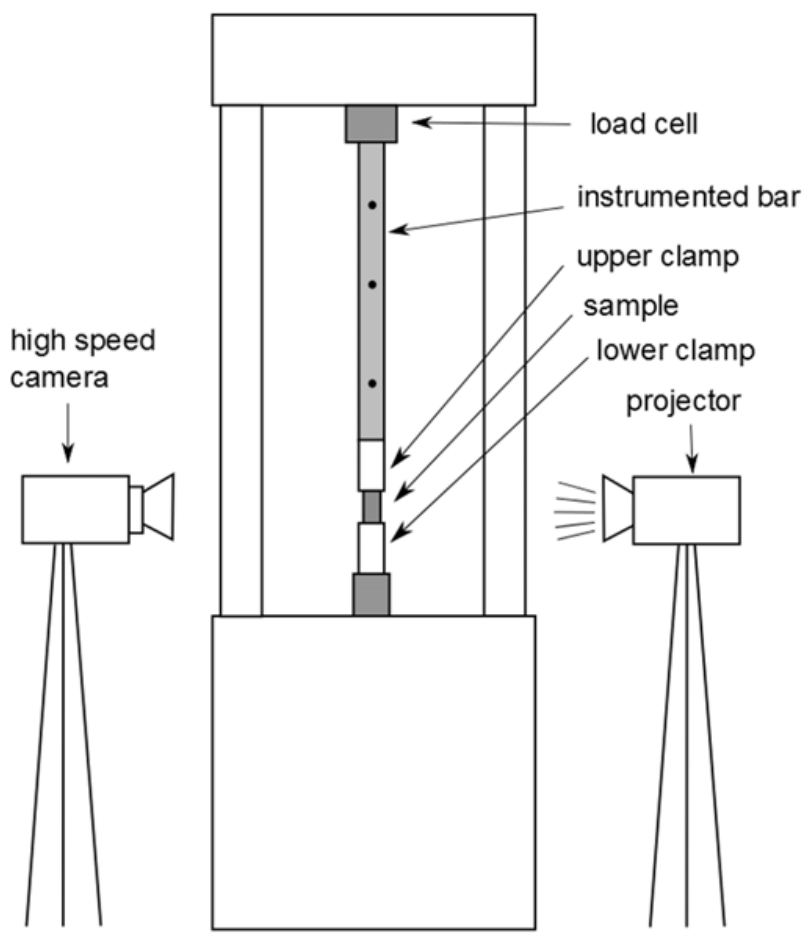

Fig. 3. Sketch representation of the experimental configuration.

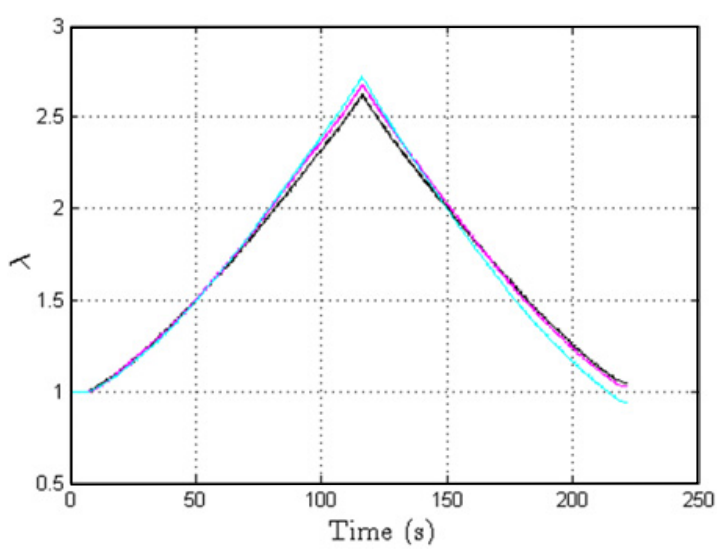

Fig. 4. Stretch ratio $\lambda$ versus time for a strain rate $\alpha=1 / \mathrm{s}$.

are shown in the figure 4. By taking the mean value of stretch ratio we calculate an average value of the stretch in the sample and hence, after smoothing and derivation, we determine the true strain rate $\alpha$ that is constant over time both during loading and unloading (see Fig. 5). It confirms that the set-up is well-designed and permits constant true strain rates experiments ranging from 0.001 to $10 / \mathrm{s}$. We also perform tests up to $100 / \mathrm{s}$ but for this velocity, unloading is not possible. Moreover, we attain the limits of the machine, we are not able to perform constant true (or nominal) strain rates experiments. However, the strain rate recorded is not so far from the value we prescribe (see Fig. 6). Furthermore, the beginning of the experiment presents a wave propagating in the specimen, which means that the strain field is not constant and so we can not prescribe constant true strain rate. So, even if we attain the limits of the machine, the strain rate can not be 


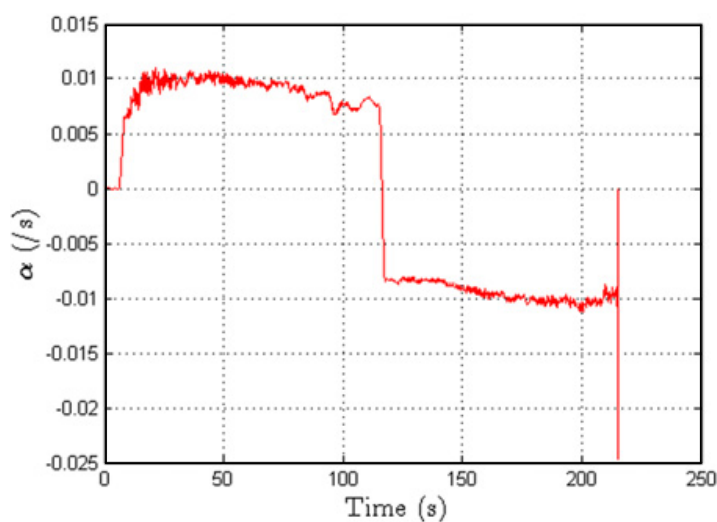

Fig. 5. Strain rate $\alpha$ versus time for a strain rate $\alpha=1 / \mathrm{s}$.

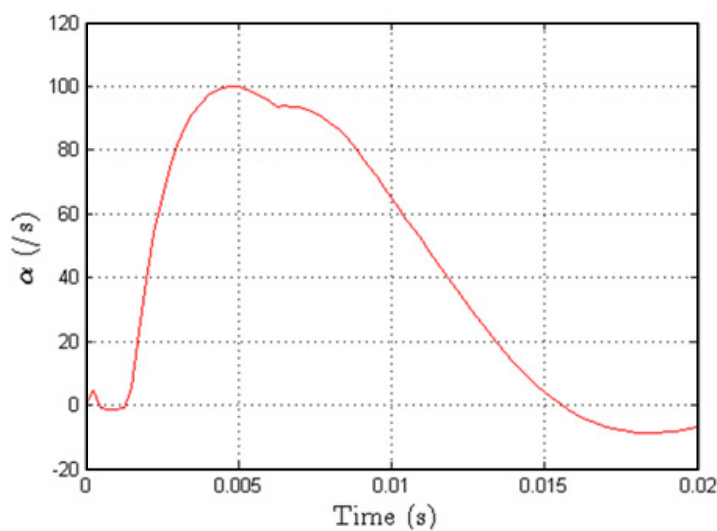

Fig. 6. Strain rate $\alpha$ versus time for a strain rate $\alpha=100 / \mathrm{s}$.

constant in the material. Nevertheless, it heads to valuable information as well, as we are able to record all the values.

\subsection{Stress}

As expected, at low strain rates, the force cell gives relevant results (see Fig. 7), but as the strain rate increases, noise and perturbation in the signal are observed (see fig. 8). On the other hand, the signal recorded by the strain gauges placed on the aluminium bar gives good results at intermediate strain rates (10-100/s) but deviates for low strain rates. This is due to the heating of the bar that occurs during the test because of the projector used to light the sample. So, for small strain rates $(0.01 / \mathrm{s})$, we only consider the force cell to compute the stress. At intermediate strain rates (10-100/s), we only consider the gauge signals. As there is no noticeable time shifting between the 3 curves, the mean value is considered instead of using the decorrelation technique.

\subsection{Stress-strain response}

Finally, filtering the stress signal over the time gives smooth curves of stress and stretch versus time. Using those, the stress-strain response figure can be drawn (Fig. 9). The tested material experiences a strengthening as the strain rate increases as it has been shown by Hoo Fatt

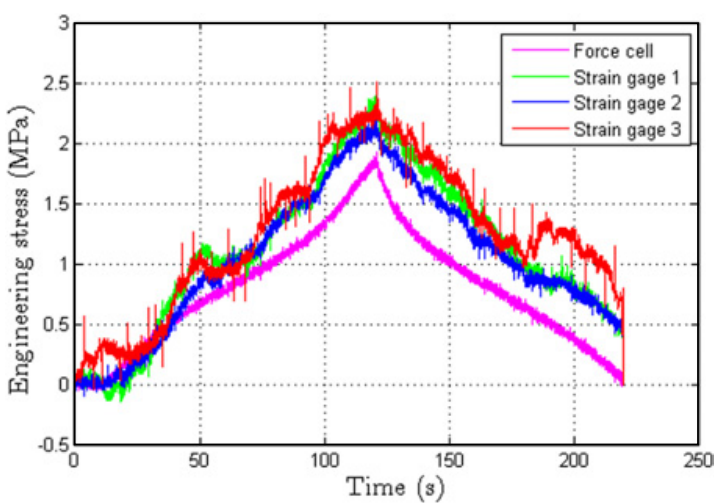

Fig. 7. Engineering stress versus time for a strain rate $\alpha=0.01 / \mathrm{s}$.

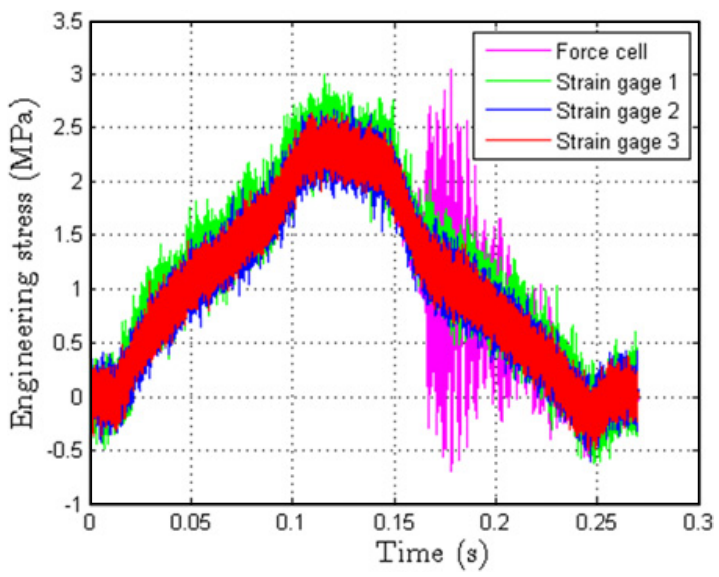

Fig. 8. Engineering stress versus time for a strain rate $\alpha=10 / \mathrm{s}$.

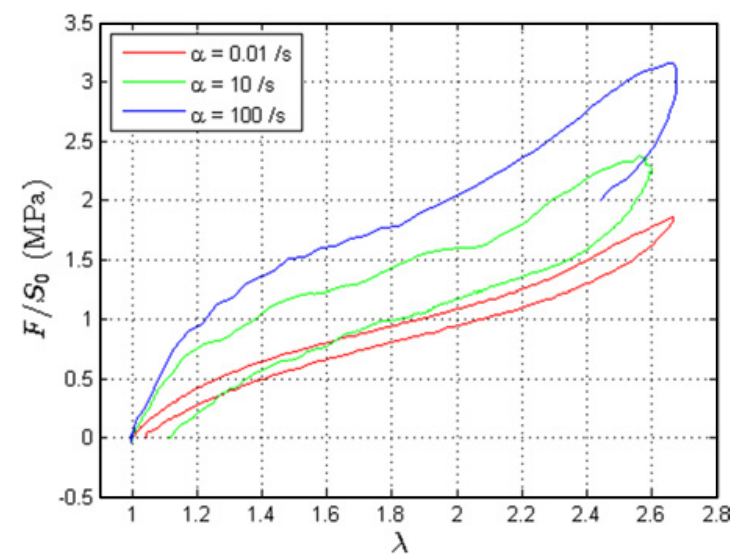

Fig. 9. Engineering stress with respect to stretch ratio for different strain rates.

\& Ouyang [5] for example. A real benefit of using a high speed machine instead of a home made machine is the fact that one can prescribe the loading signal. In our case, we were able to load and unload the specimen at desired strain rates except at 100/s where unloading is too risky. Such tests give an extra information about the behaviour of the material: the dissipation. It is important to notice that it evolves with strain rate. Indeed, the hysteresis loop is larger for high strain rates (10-100/s), showing that the dissipation increase with the strain rate in the range from 0.01 to $10 / \mathrm{s}$ (Fig. 10). 


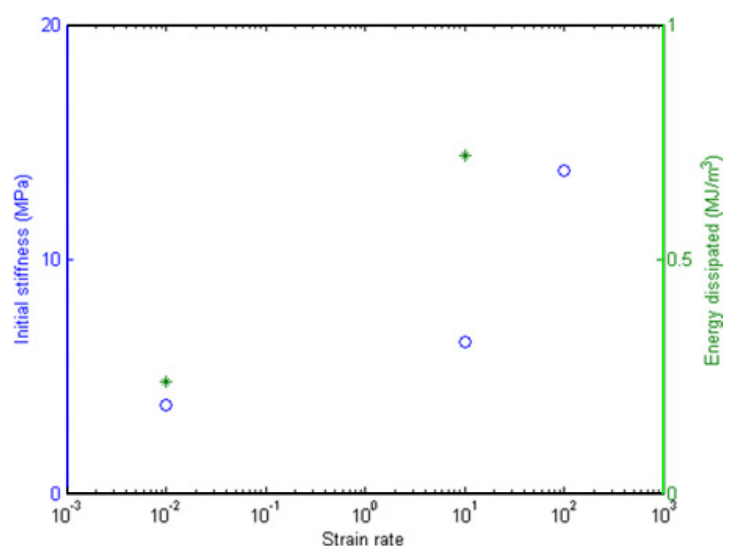

Fig. 10. Dissipation (o) and initial stiffness (*) depending on the strain rate.

\section{Conclusion}

We have presented a method to perform tests at constant true strain rate. This method necessitates the use of a special sample with a constant section, between the clamps, to ensure homogeneous strain and stress fields. A tracking point technique enables to measure the strain in the sample. For the stress measurement, weconsider two types of sensors: (i) a classical force cell for low strain rates, (ii) a three-strain-gauges tubular aluminum bar for the intermediate strain rates. This method permits to perfectly prescribe the strain history for strain rates up to 10/s. Above this speed, we attain the limits of the machine, but also of the material: the strain field is no longer constant at the beginning, for strain of some percents. Nevertheless, we determine the stretch-stress-time curve for the material during the loading of the material but also during the unloading (up to $10 / \mathrm{s}$ ). We also compute the energy dissipated over 1 cycle. The results show that SBR experiences a strengthening as the strain rate increases and a change in the hysteresis loop. However, it would be interesting to compare those results with tests performed at constant nominal strain rate.

\section{Acknowledgements}

The authors would like to thank P. Guégan and F. Pasco (Ecole Centrale de Nantes) for their help in the experimental work.

\section{References}

1. J. Yi, M. Boyce, G. Lee, and E. Balizer, Polymer 47(1), (2006) 319-329.

2. S. S. Sarva, S. Deschanel, M. C. Boyce, and W. Chen, Polymer 48(8), (2007) 2208-2213.

3. G. Gary, Techniques de l'Ingénieur BM 7 (176) (2001) $1-10$.

4. M. S. Hoo Fatt and I. Bekar., Journal of Materials Science 39(23), (2004) 6885-6899.

5. M. S. Hoofatt and X. Ouyang, Mechanics of Materials 40(1-2), (2008) 1-16.

6. C.M. Roland, J.N. Twigg, Y. Vu, and P.H. Mott, Polymer 48(2), (2007) 574-578.

7. R. Othman, P. Guéguan, G. Challita, F. Pasco, D. Lebreton, Int J Impact Eng 36 (2009) 460-467.

8. R. Othman, M.N. Bussac, P. Collet, G. Gary, C. R. Acad. Sci. Série IIb 329 (2001) 369-376.

9. M. N. Bussac, P. Collet, G. Gary, R. Othman, Journal of the Mechanics and Physics of Solids 50 (2002) 321-349.

10. R. Othman, G. Gary, Exp Mech 47 (2007) 295-299.

11. J. Shim, D. Mohr, Int J Impact Eng 36 (2009) 11161127.

12. S. Aloui, R. Othman, A. Poitou, P. Guégan, S. ElBorgi, Mech Res Comm 35 (2008) 236-244.

13. R. Othman, S. Aloui, A. Poitou, Polym Test 29 (2010) 616-623. 